\title{
NF-kB p65 repression by the sesquiterpene lactone, Helenalin, contributes to the induction of autophagy cell death
}

Chuan Bian Lim', Pan You Fu ${ }^{3}$, Nung Ky ${ }^{1}$, Hong Shuang Zhu' ${ }^{1}$ XiaoLing Feng ${ }^{2}$, Jinming Li ${ }^{1}$, Kandhadayar Gopalan Srinivasan ${ }^{4}$, Mohamed Sabry Hamza ${ }^{5 *}$ and Yan Zhao ${ }^{1 *}$

\begin{abstract}
Background: Numerous studies have demonstrated that autophagy plays a vital role in maintaining cellular homeostasis. Interestingly, several anticancer agents were found to exert their anticancer effects by triggering autophagy. Emerging data suggest that autophagy represents a novel mechanism that can be exploited for therapeutic benefit. Pharmacologically active natural compounds such as those from marine, terrestrial plants and animals represent a promising resource for novel anticancer drugs. There are several prominent examples from the past proving the success of natural products and derivatives exhibiting anticancer activity. Helenalin, a sesquiterpene lactone has been demonstrated to have potent anti-inflammatory and antitumor activity. Albeit previous studies demonstrating helenalin's multi modal action on cellular proliferative and apoptosis, the mechanisms underlying its action are largely unexplained.
\end{abstract}

Methods: To deduce the mechanistic action of helenalin, cancer cells were treated with the drug at various concentrations and time intervals. Using western blot, FACS analysis, overexpression and knockdown studies, cellular signaling pathways were interrogated focusing on apoptosis and autophagy markers.

Results: We show here that helenalin induces sub-G1 arrest, apoptosis, caspase cleavage and increases the levels of the autophagic markers. Suppression of caspase cleavage by the pan caspase inhibitor, Z-VAD-fmk, suppressed induction of LC3-B and Atg12 and reduced autophagic cell death, indicating caspase activity was essential for autophagic cell death induced by helenalin. Additionally, helenalin suppressed NF-kB p65 expression in a dose and time dependent manner. Exogenous overexpression of p65 was accompanied by reduced levels of cell death whereas siRNA mediated suppression led to augmented levels of caspase cleavage, autophagic cell death markers and increased cell death.

Conclusions: Taken together, these results show that helenalin mediated autophagic cell death entails inhibition of NF-KB p65, thus providing a promising approach for the treatment of cancers with aberrant activation of the NF-KB pathway.

Keywords: Helenalin or Hele(Helenalin), Autophagy, Caspase, NF-KB, Atg12 and LC3-B

\footnotetext{
*Correspondence: sabry.hamza@merck.com; zhaoyan@ntu.edu.ag

${ }^{1}$ Division of Chemical Biology and Biotechnology, School of Biological

Sciences, College of Science, Nanyang Technological University, 60 Nanyang

Drive, Singapore 637551, Singapore

${ }^{5}$ MSD, Translational Medicine Research Center, 8 Biomedical Grove, \#04-01/

-05 \& \#05-01/05, Neuros Building, Singapore 138665, Singapore

Full list of author information is available at the end of the article
} 


\section{Background}

The efforts of many researchers during the past dozen years to identify novel compounds with anticancer activity have pointed to plants and herbs used in herbal medicine. The rationale behind this approach is that herbal medicine looks back on a 5000-years tradition. Hence, it can be expected that many medicinal herbs and plants have been selected for pharmacological activity [1]. Many studies have shown that herbal medicine is indeed a valuable resource for novel compounds with activity against tumors in vitro and in vivo [2-4]. Hence, the chances to find novel compounds with activity against tumor cells in natural product libraries are higher than in synthetic libraries.

In this regard, helenalin, a naturally occurring sesquiterpene lactone has generally been considered as a distinctly promising and potent antitumor compound. Helenalin has been shown to be a potent inhibitor of hTERT (human Telomerase Reverse Transcriptase) and telomerase in hematopoietic cancer cell [5], induces apoptosis in activated CD4+ T cells through the mitochondrial apoptosis pathway [6] and have been shown to selectively alkylate the p65 subunit of NF-kB [7].

In this report, we provide a mechanism by which NF- $\mathrm{kB}$ p 65 plays a significant role in modulating autophagy induced cell death by the sesquiterpene lactone, helenalin. NF-kB p65 expression is down regulated upon helenalin treatment in a time and dose dependent manner. Down regulation of NF- $\mathrm{kB}$ p65 in turn induces caspase cleavage and autophagic genes Atg12 and LC3-B resulting in sub-G1 arrest and cell death. Exogenous expression of NF-kB p65 attenuates caspase cleavage and subsequently autophagy, demonstrating a mechanistic pathway of helenalin induced autophagic cell death. siRNA mediated transcriptional knockdown of NF-kB p65, Atg12 or LC3-B or inhibition of caspase cleavage using Z-VADfmk diminishes autophage cell death. In addition, helenalin induced apoptosis by activating the intrinsic apoptosis pathway. Taken together, we surmise that helenalin mediated apoptotic and autophagic cell death may provide a promising treatment strategy for cancers with aberrant activation of the NF-kB pathway.

\section{Methods}

\section{Cell Culture and drug treatment}

A2780 (human ovarian cancer cell line), RKO (colon carcinoma cancer cell line) and MCF-7 (breast adenocarcinoma cancer cell line) were obtained from ATCC (Manassas, VA). Cells were cultured in Dulbecco's modified Eagle's medium, supplemented with $10 \%$ fetal bovine serum, $1 \%$ penicillin-streptomycin (all from GIBCO $^{\circledR}$ Invitrogen, Carlsbad, CA) in a humidified $5 \% \mathrm{CO} 2$ atm at $37{ }^{\circ} \mathrm{C}$. Cells were treated with helenalin (Ambrosa-2,11 (13)-dien-12-oic acid, 6- $\alpha, 8$ - $\beta$-dihydroxy-4-oxo-, 12,8- lactone) purchased from EMD biosciences (Gibbstown, NJ). Dimethyl sulfoxide was used throughout the experiments as the vehicle control. At least three biological experiments were performed to verify observations.

\section{Flow cytometry analysis}

Cells were harvested after drug treatment and fixed with $70 \%$ ethanol. Fixed cells were treated with RNase $(100 \mu \mathrm{g} / \mathrm{ml})$ and stained with propidium iodide $(50 \mu \mathrm{g} / \mathrm{ml})$. Subsequently, stained cells were analyzed for DNA content by flow cytometry using FACScalibur (Becton Dickinson, Franklin Lakes, NJ). Cell cycle fractions were quantifies using the CellQuest software (BD Biosciences, San Jose, CA). Further details can be found in [8]. At least three biological experiments were performed to verify observations.

\section{Cell Proliferation Assay}

Inhibition of cell proliferation by helenalin was assessed using the MTT assay (Roche, Indianapolis, IN). Briefly, A2780, MCF-7 or RKO cells were plated in 96-well culture plates $\left(5 \times 10^{4}\right.$ cells/well $)$ and treated the following day with helenalin or DMSO vehicle as described in the results section. Following helenalin treatment, cells were incubated with MTT labeling reagent for $4 \mathrm{~h}$, solubilized in $10 \%$ SDS, and the MTT metabolite formazan crystals were quantitated at $575 \mathrm{~nm}$ on a microplate reader (Tecan, Männedorf, Switzerland). All experiments were performed and verified using at least three biological replicates.

\section{Clonogenic Assay}

To determine the growth suppression effect of helenalin treatment, A2780 cells were treated with helenalin or DMSO vehicle for $24 \mathrm{~h}$. After treatment, cells were replated in complete DMEM and allowed to grow for 14 days to form colonies that were then stained with crystal violet (Sigma) and quantified. All experiments were performed and verified using at least three biological replicates.

\section{Annexin-V measurements}

Direct fluorescence staining of apoptotic cells for flow cytometric analysis was performed with the Annexin V-FITC apoptosis detection kit (BD Pharmingen, San Jose, CA). After the indicated times, cells were harvested and stained according to the manufacturer's protocol. Stained cells were analyzed in a flow cytometer. All experiments were performed and verified using at least three biological replicates.

\section{Western Blotting}

Western blotting procedure was followed according to [8]. Briefly, cells were lysed in appropriate volume of lysis buffer (Sigma Aldrich, St. Louis, MO). $50 \mu \mathrm{g}$ of protein samples were separated by SDS-PAGE and transferred onto nitrocellulose membrane (Bio-Rad, Hercules, 
CA). The membranes were immunoblotted with primary antibodies purchased from Cell Signal Technology (Danvers, MA) or Santa Cruz Biotechnology, Inc (Santa Cruz, CA). Blots were incubated with horseradish peroxideconjugated goat anti-rabbit, goat anti mouse or rabbit anti-goat secondary antibodies purchased from Santa Cruz Biotechnology, Santa Cruz, CA. All experiments were performed and verified using at least three biological replicates.

\section{siRNA transfection}

A2780 cells were transfected with non-targeting control siRNA (siRNA Neg), siRNA Atg12, siRNA LC3-B or siRNA RelA p65 when cells reached $80 \%$ confluency. After $24 \mathrm{~h}$, cells were split 1:3, and treated with helenalin or DMSO the next day. Final siRNA concentration was $100 \mathrm{nM}$ and transfection was performed using Lipofectamine RNAimax (Invitrogen, Carlsbad, CA) according to the manufacturer's protocol. Target sequences used for siRNA against Atg12, $L C 3-B$ and RelA p65 were 5'CUUAACAGAUGUGAUCUAU-3', 5'-GUAAU UCCAGCAGUAAUUU-3', 5'-CUCAAGAUCUGCCGA GUGA-3' respectively. All experiments were performed and verified using at least three biological replicates.

\section{Plasmid transfection}

A2780 cells were transfected with 2.0 ug of empty vector or NF-kB RelA p65 overexpressing vector (purchased from Origene Technologies, Rockville, MD; Cat \# RC220780) using FuGENE 6 transfection reagent (Roche, Indianapolis, IN) following manufacturer's instructions. All experiments were performed and verified using at least three biological replicates.

\section{Acridine Orange staining for autophagy detection}

Cell staining with Acridine orange $(10 \mathrm{mg} / \mathrm{ml}$ in water, A8097, Sigma Chemical Co) was performed according to published procedures [9], adding at a final concentration of $1 \mathrm{mg} / \mathrm{ml}$ for a period of $15 \mathrm{~min}$. Bafilomycin A1 (Sigma Chemical Co.) was dissolved in DMSO and added to the cells $45 \mathrm{~min}$ before addition of acridine orange. Photographs were obtained with a fluorescence microscope and percent of staining was determined by harvesting cells by trypsinization and measuring with a FACSCalibur from (Becton Dickinson) using CellQuest software. All experiments were performed and verified using at least three biological replicates.

\section{Results and Discussion}

\section{Helenalin Inhibits Cell Proliferation and Clonogenic} Survival in cancer cells

To examine the effect of helenalin on cell proliferation and clonogenic survival, human ovarian cancer A2780 cells were treated with helenalin and effects on cell proliferation and survival was determined using phase contrast microscopy, crystal violet staining and MTT assays. Phase contrast micrographs of A2780 cells treated with increasing concentrations of helenalin of 0.5 , 1.0 and $2 \mathrm{uM}$ for $24 \mathrm{~h}$ showed changes in cell number and morphology. Morphologic signs of apoptosis included changes such as membrane blebbing and apoptotic body formation (Figure 1A). To measure the amount of cell survival after helenalin exposure, A2780 cells were cultured in the presence of increasing doses of helenalin (serial dilution for drug concentration ranging from $10 \mathrm{uM}$ to $0.001 \mathrm{uM}$ ) for $24 \mathrm{~h}$ and cell survival was determined using the MTT assay. As shown in Figure 1B, increasing concentrations of helenalin reduced the percentage of cell survival in a dose dependent manner. To further investigate the growth suppression effect of helenalin, we performed in vitro clonogenic assays. Figure $1 \mathrm{C}$ shows the effects of helenalin on the clonogenic potential of the control (DMSO) and helenalintreated A2780 cells. Helenalin reduced clonogenicity of A2780 cells in a dose-dependent manner, where $2 \mathrm{uM}$ of helenalin completely suppressed clonogenic growth.

\section{Induction of G1 Phase Arrest and cell death by Helenalin}

To identify the mechanism of helenalin-induced cell proliferation inhibition and cell death, we examined the effects of helenalin on cell cycle distribution by flow cytometry. As shown in Figure 2A, cells in sub-G1 phase increased in a helenalin dose dependent manner, analogous with the earlier observation that helenalin inhibited cell proliferation (Figure 1A). As much as $25 \%$ of cells in sub-G1 were observed in A2780 cells treated with $2 \mathrm{uM}$ helenalin (Figure $2 \mathrm{~B}$ ). To rule out that our findings were cell line specific, we replicated our experiments in MCF-7 breast adenocarcinoma cancer cell line and RKO colon carcinoma cancer cell lines. As shown in Additional file 1: Figure S1 A and C, increase in sub-G1 levels were observed in these additional cancer cell lines, with the RKO cell line exhibiting greater sensitivity to helenalin whilst MCF-7 cells were comparatively less sensitive. As demonstrated previously for the A2780 cancer cell line (Figure 1B), helenalin also reduced the percentage of cell survival in a dose dependent manner in both the MCF-7 and RKO cancer cell lines (Additional file 1: Figure S1 B and D). As sub-G1 levels are an indicative measurement of cell death $[10,11]$, we examined whether the fraction of cells in sub-G1 after helenalin treatment was attributable to cells undergoing apoptosis. We measured apoptotic cell death by staining with FITC-Annexin V and Propidium Iodide and performed flow cytometry to analyze sub fractions of cells undergoing apoptosis or necrosis. As revealed in Figure 2C, partial increase in apoptotic cells was observed after helenalin treatment when compared to 


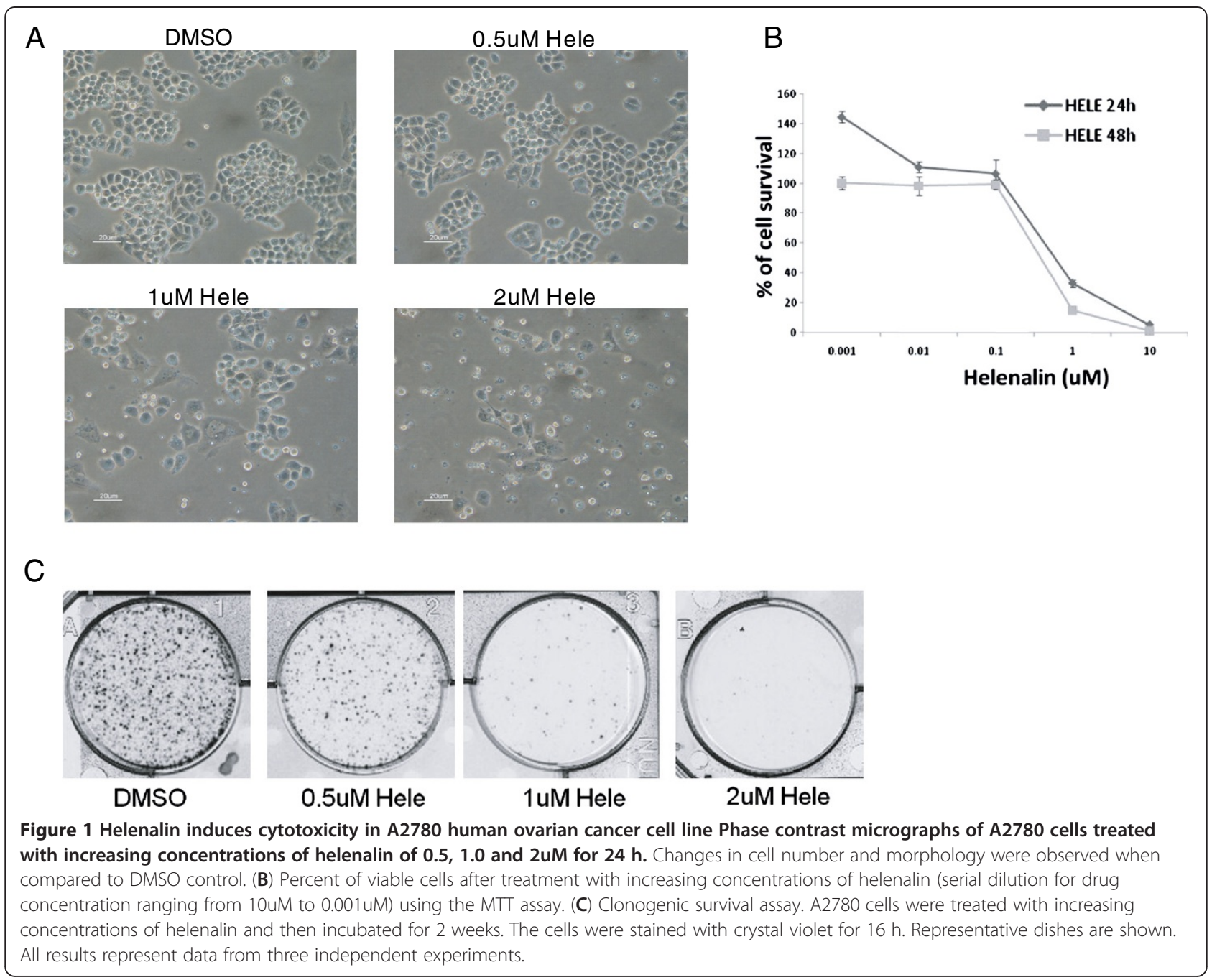

the FACS data (Figure 2A,B), suggesting cells in sub-G1 undergo cell death via apoptosis in addition to other cell death mechanisms. In addition to the dose dependent effects of helenalin observed, we performed additional experiments to investigate the effects on A2780 cells exposed to helenalin at varying treatment times. Flow cytometry assays performed on cells harvested after different exposure times demonstrate an increase in sub-G1 levels with increasing exposure to helenalin (Figure 3A). As much as $35 \%$ of cells are in sub-G1 $24 \mathrm{~h}$ post treatment with helenalin (Figure 3B).

\section{Helenalin induces cell death via caspase cleavage and induction of autophagy}

To further investigate the mechanistic action of cell death induced by helenalin, we performed western blot analysis to detect proteins that have been shown to be involved in both the intrinsic and extrinsic apoptosis pathways. Cells treated with increasing concentrations of helenalin were lysed and subjected to western blot analysis for cleaved caspases 3 and 9 and also for cleaved PARP. After $24 \mathrm{~h}$ of treatment, the levels of cleaved caspases increased with increasing concentrations of helenalin (Figure 4A). Using a dose of $2 \mathrm{uM}$ helenalin, it was observed that levels of cleaved caspase 3, 9 and PARP were detected at the outset of $8 \mathrm{~h}$ post treatment with subsequent increase in cleavage with protracted treatment times (Figure 4B). To substantiate the prerequisite of caspase cleavage as a determinant for helenalin induced cell death, we employed the use of the pan caspase inhibitor, Z-VAD-fmk to block caspase cleavage during helenalin treatment and determined the levels of sub-G1cells by flow cytometry. Addition of Z-VAD-fmk to cells prior to helenalin treatment suppressed caspase 3, 9 and PARP cleavage (Figure 4C) and levels of sub-G1 cells measured by flow cytometry showed comparable levels to those of control treated cells versus to those of cells treated 
A
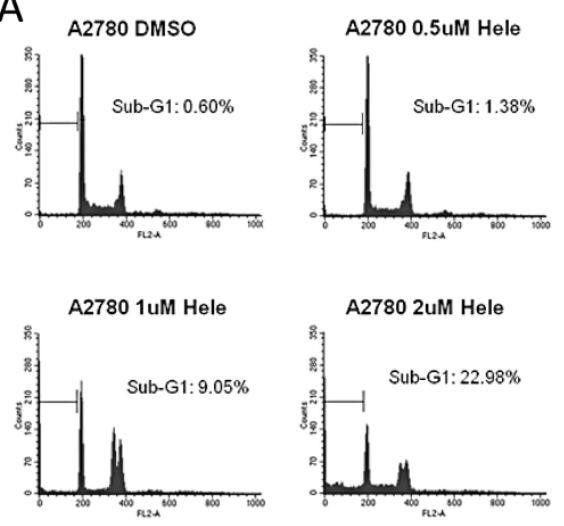

C
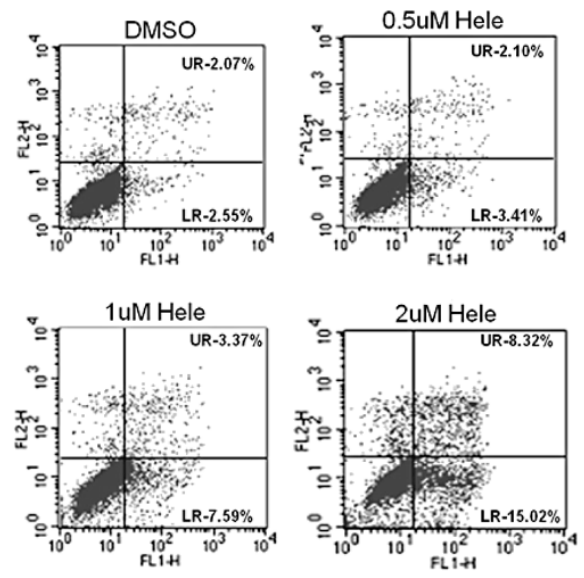

B

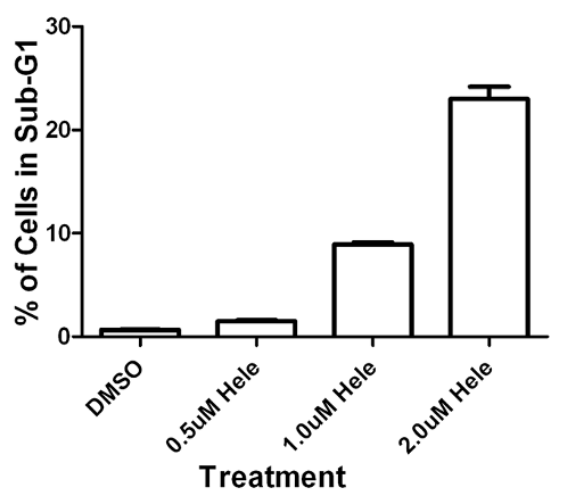

D

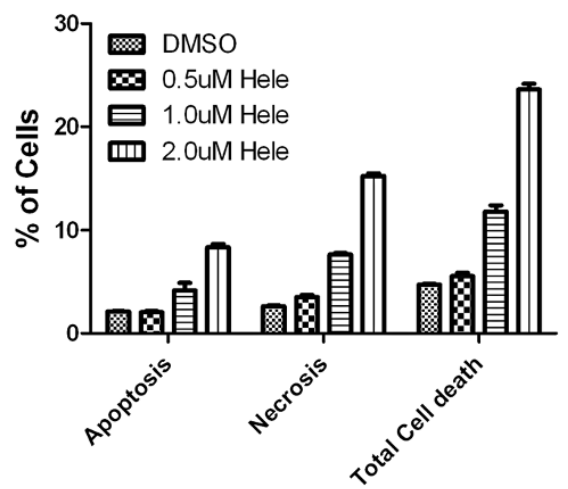

Figure 2 Helenalin induces cell cycle arrest in G1 phase and modulates apoptosis in a dose-dependent manner. (A) $1.85 \times 105 \mathrm{cells} / \mathrm{ml}$ were seeded onto 6-well plates and incubated for $24 \mathrm{~h}$. Various concentrations of helenalin were added to the culture medium and incubated for an additional $24 \mathrm{~h}$. Cells were then harvested and analyzed by flow cytometry. The cell cycle phase distribution was determined using CellQuest software. (B) Percent of cells in Sub-G1 after drug treatment representing three independent experiments. (C) Measurement of apoptotic cell death by staining with FITC-Annexin V and Propidium lodide. Lower right hand quadrant for each dose treatment represents percent of apoptosis, while upper right hand quadrant represents early necrotic cells. (D) Percent of cells that are either apoptotic, necrotic or total cell death as measured by staining cells with FITC-Annexin V and Propidium lodide. Percentages are the average of three independent biological replicates.

with helenalin alone (Figure 4D). Quantitative measurements of cells in sub-G1 were reduced from levels of $\sim 25 \%$ in helenalin alone treated cells to less than $2 \%$ with helenalin in combination with Z-VAD-fmk (Figure 4E). We subsequently investigated the intrinsic cell death pathway by assessing the protein levels of Bcl-2, Bax and Bid in lysates from cells treated with different concentrations of helenalin. As shown in Figure 5A, no appreciable differences in protein expression were observed suggesting that helenalin induced cell death was not attributable to activation of the Bcl2, Bax and Bid. Interestingly, as shown in Figure 4, helenalin activated caspase 9, strongly suggesting helenalin induces intrinsic apoptotic cell death. We next investigated the levels of Atg12 and LC3-B, both biomarkers indicative of autophagy cell death. As demonstrated in Figure 5B, there was a dose dependent increase in protein levels of Atg12 and LC3-B with increasing concentrations of helenalin. Our findings are in contrast to recently published data where there was no increase of LC3-B detected in Jurkat cells treated with helenalin [12]. The authors of this manuscript can only speculate to the differences in observations as cell line specific. To verify that cells were indeed undergoing autophagy, cells were treated with varying concentrations of helenalin and stained with Acridine Orange solution to detect and measure acidic vesicular organelle (AVO) formation. As shown in Figure 5C, vital staining of cells with acridine orange showed the accumulation of AVO in the cytoplasm of cells exposed to increasing concentrations of helenalin. This was inhibited by addition of bafilomycin A1 (200 nM), an $\mathrm{H}^{+}$ATPase 


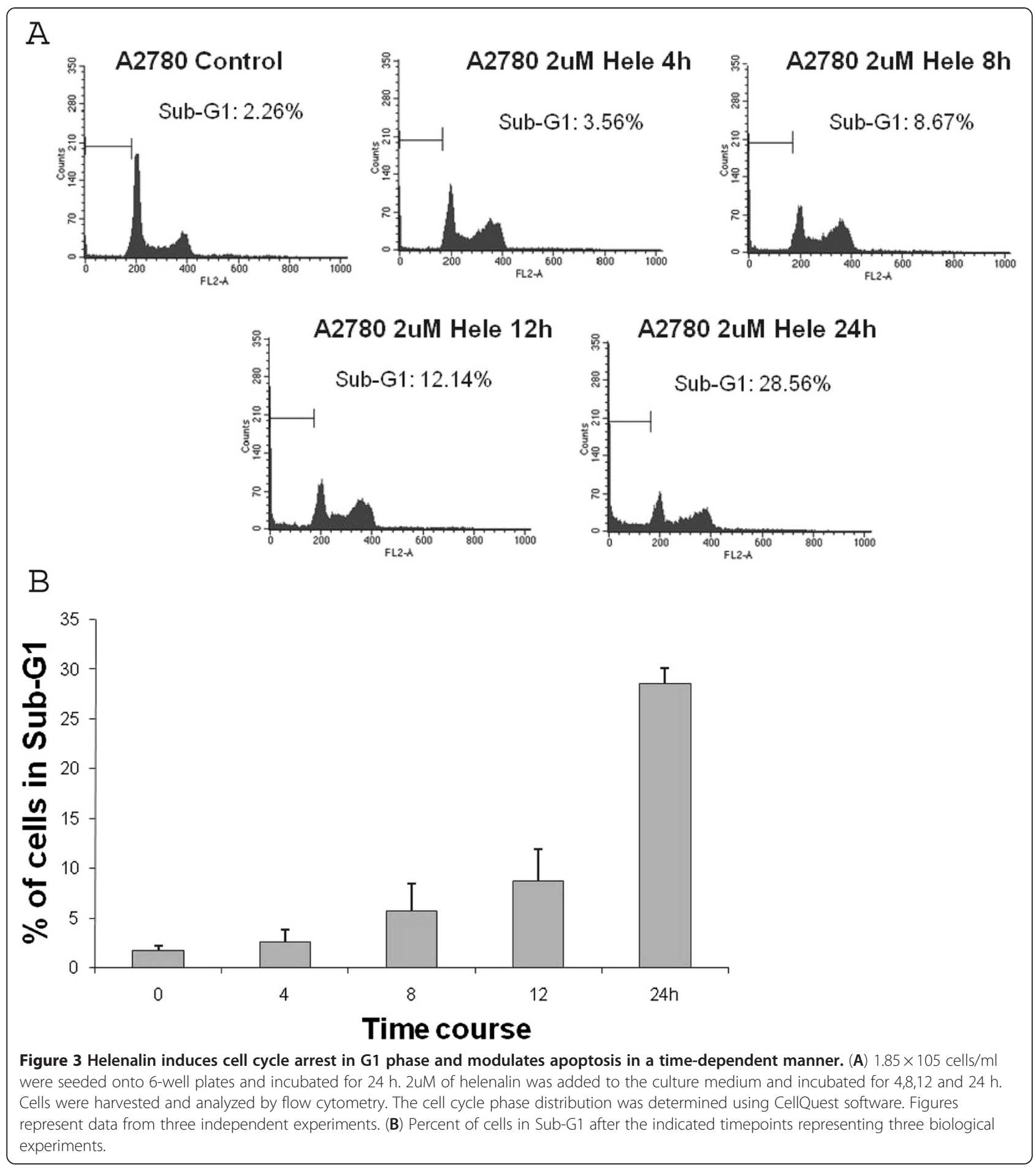

inhibitor (Figure 5C). The amount of AVO staining was quantitated in cells treated with helenalin or/and Balifomycin A1, by trypsinizing and harvesting cells for FACS analysis. Approximately $80 \%$ of cells treated with $2 \mathrm{uM}$ helenalin for $24 \mathrm{~h}$ were positive for AVO staining and these levels were completely abrogated by the addition of Bafilomycin A1 (Figure 5D).
Inhibition of Atg12 and LC3-B expression reduces caspase cleavage and cell death induced by Helenalin

To investigate the significance of Atg12 and LC3-B in cells undergoing helenalin induced autophagy, we depleted Atg12 and LC3-B in A2780 cells using siRNAmediated knockdown. Post siRNA transfection and 


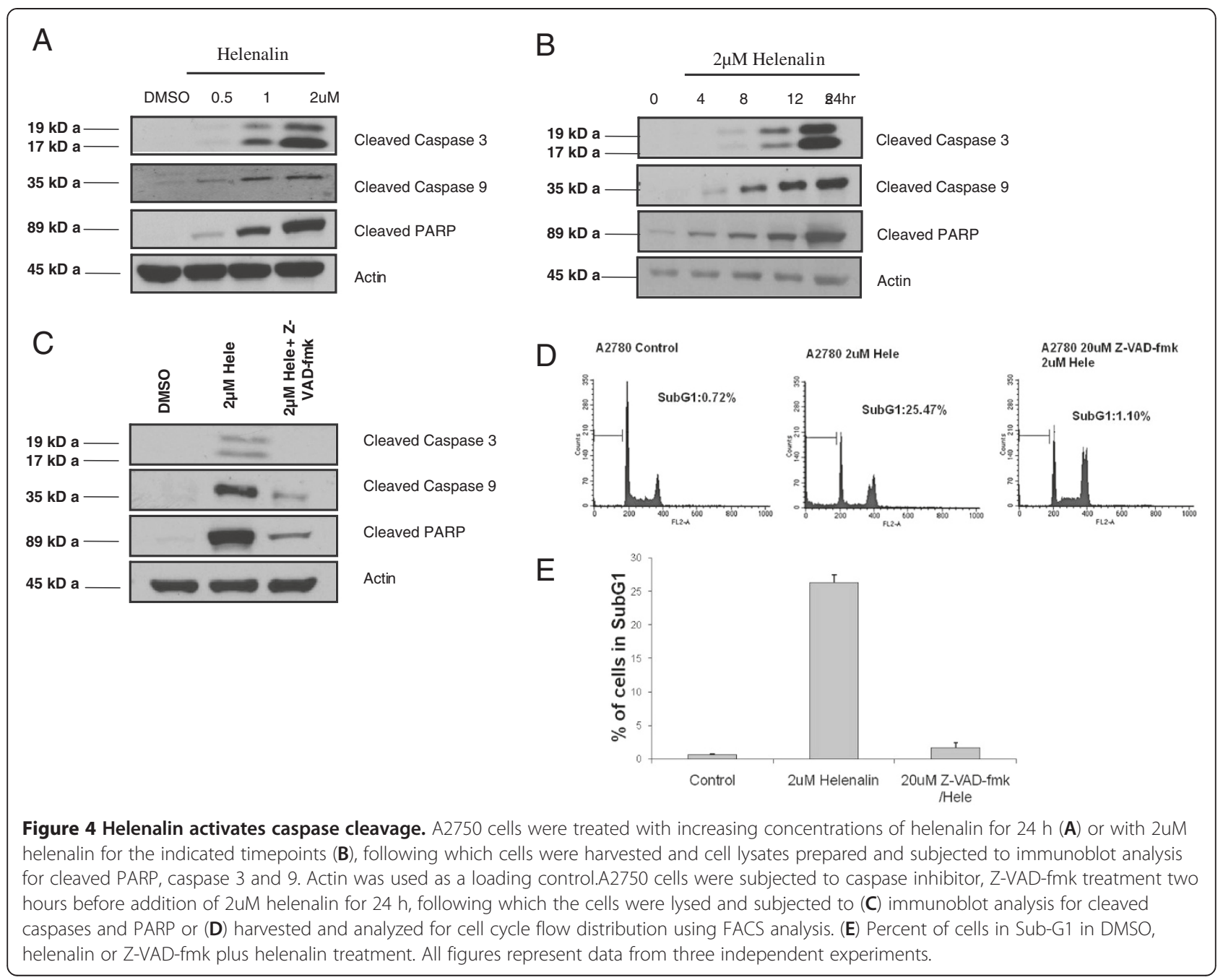

upon helenalin treatment, we observed a reduction of protein levels for both Atg12 and LC3-B when compared to cells treated with a non targeting control siRNA (Figure 6A and B). Intriguing, upon helenalin treatment, the protein levels of cleaved caspases were reduced in cells depleted of Atg12 and LC3-B as compared to cells treated with helenalin and transfected with a control non-targeting siRNA (Figure 6A and B). In addition, flow cytometry analysis performed in cells treated with Atg12 or LC3-B showed a decrease in sub-G1 levels when compared to cells treated with a non-targeting siRNA (Figure $6 \mathrm{C}$ and $\mathrm{D}$ ). This result is consistent with previous findings where decrease in LC3-B was associated with reduced autophagy and cells treated with LC3-B or Beclin 1 siRNA inhibited caspase-3/8 activation [13]. In the context of helenalin induced cell death, this result implies that both Atg12 and LC3-B modulate caspase cleavage essential for autophagy.
NF-KB p65 inhibition by Helenalin is essential for caspase cleavage and induction of autophagy

To ascertain the mechanism by which helenalin induces Atg12 and LC3-B expression, we concerted our efforts in understanding the role of the transcription factor NF-kB p65 in helenalin induced autophagy. Previous reports have demonstrated helenalin's role in anticancer and anti-inflammatory effects by inhibiting NF$\kappa \mathrm{B}$ and telomerase activity and impairing protein and DNA synthesis [6]. In addition, helenalin interacts with RelA to inhibit DNA binding to its cognate response elements and by inhibiting activation of the transcription factor NF-kB [7]. Blockade of NF-kB/p65 binding to DNA with helenalin correlated with induction of cell death in a dose-dependent manner [14]. Numerous reports have demonstrated NF- $\mathrm{kB}$ p65 to play a role in autophagy induced cell death $[13,15]$, however the function in which helenalin participated in autophagy was unknown. Amalgamating this information, we 


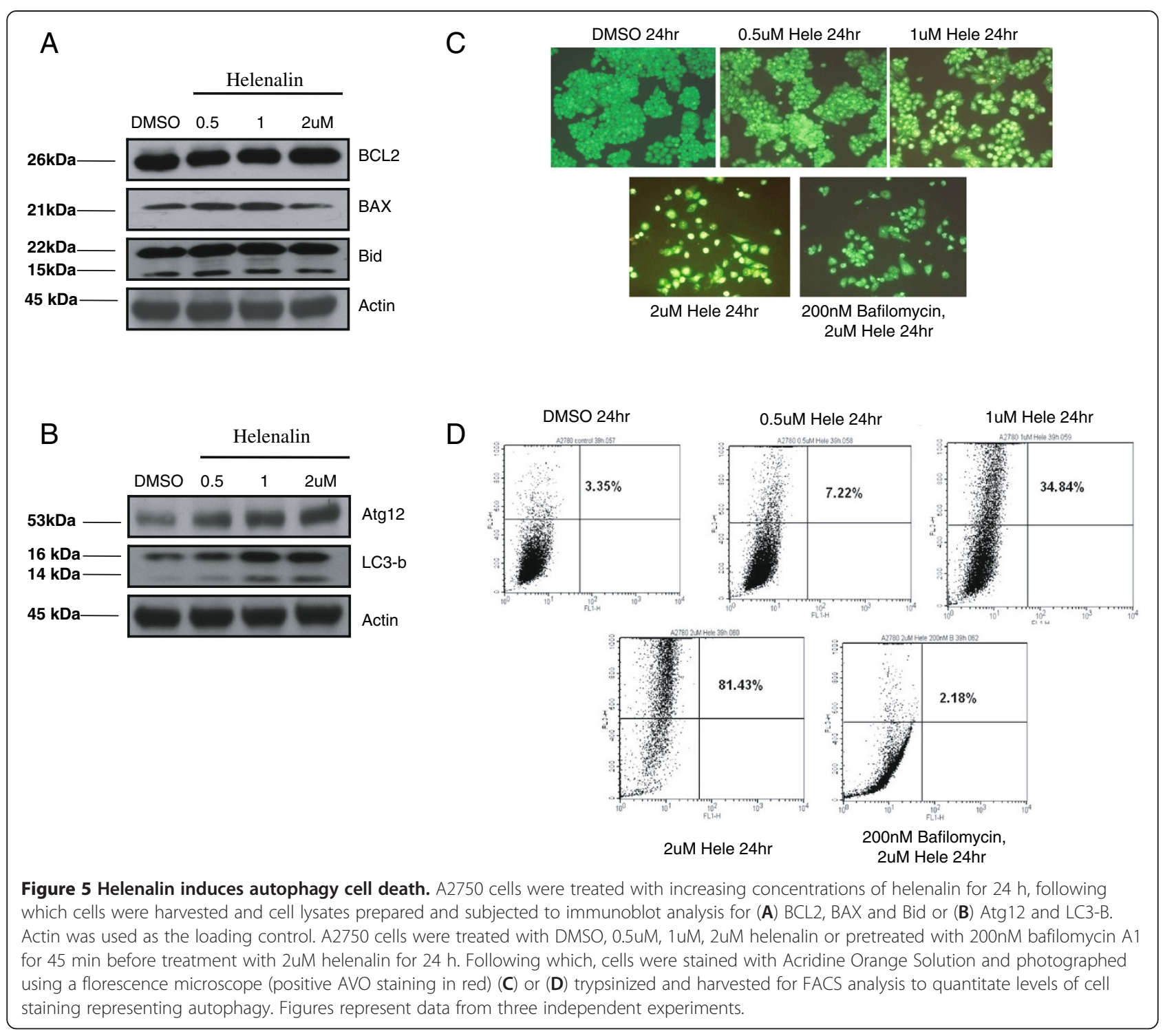

decided to investigate NF-kB p65 for its role in helenalin induced autophagy cell death. As shown in Figure $7 \mathrm{~A}$, helenalin reduced the expression of NF-kB p65 in a dose dependent manner. Exogenous overexpression of NF-kB p65 reduced the levels of cleaved caspase 3, 9 and LC3-B in cells treated with helenalin (Figure 7B) with subsequent reduction of sub-G1 levels (Figure 7C), while siRNA mediated transcriptional knockdown of NF-kB p65 increased cleaved caspase 3 and 9 but not LC3-B after helenalin treatment (Figure 7D) with consequent increase in sub-G1 levels in cells (Figure 7E). No differences in LC3-B levels were observed after siRNA knockdown of NF-kB, since after helenalin treatment, NF- $\mathrm{KB}$ levels are reduced by the drug itself. This result was recapitulated in MCF-7 and RKO cells with analogous outcomes (data not shown).

\section{Conclusions}

Helenalin induces autophagy cell death via suppression of NF-kB p65

Pharmacologically active natural compounds such as those from marine and terrestrial plants and animals represent a promising resource for novel anticancer drugs. There are several prominent examples from the past proving the success of natural products and derivatives exhibiting anticancer activity. This includes the Vinca alkaloids from Catharanthus roseus, the terpene paclitaxel from Taxus brevifolia, the DNA topoisomerase I inhibitor camptothecin from Camptotheca acuminata, and the semisynthetic derivatives etoposide and teniposide of the lignan podophyllotoxin from Podophyllum peltatum. Natural products alone or synthetics developed based on knowledge gained from natural products 


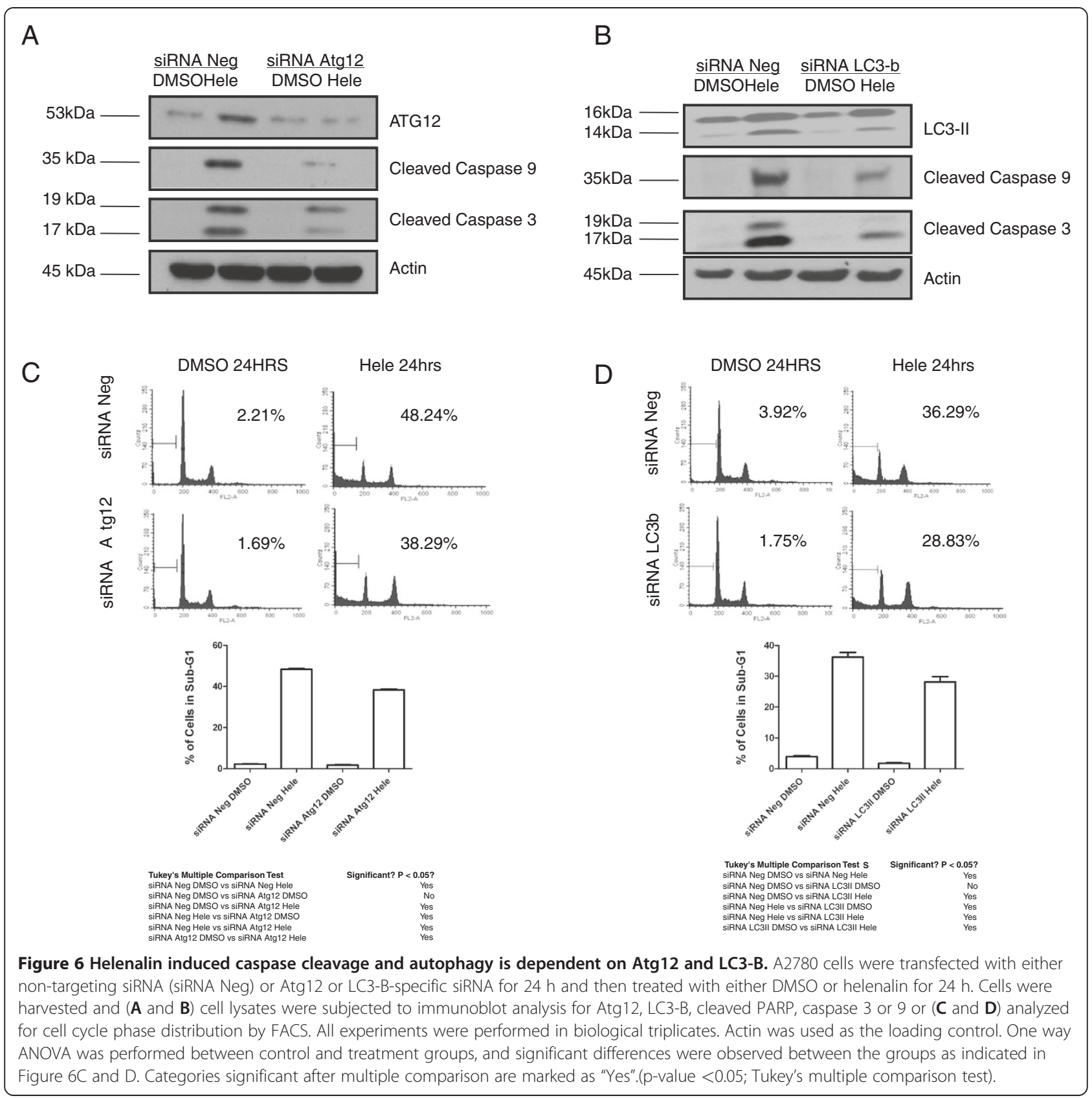

account for about $70 \%$ of anticancer therapeutics approved between 1980s and 2002 [16].

Herbal medicine has been applied in the clinic for thousands of years in Asian countries such as China, Japan and Korea [17]. However, because of its complicated chemical composition and lack of concrete evidence of its biological activity, herbal medicine is still not widely accepted by the Western medical community [18]. Unlike modern drugs in the form of a single active compound, herbal medicine is usually prepared from aqueous extracts of a few herbs and contains hundreds or even thousands of different compounds [19].
However, only a few compounds are responsible for the pharmacological effects [19]. Furthermore, the bioactive components are generally present at low level. Some components are useless or even toxic. Thus, systematic characterization of active chemicals in herbal medicinal preparations and their mechanisms of action are important for providing the rationale for their efficacy and for transforming herbal medicine practices into evidencebased medicine.

Helenalin, an extracted component of Arnica Montana and Arnica chamissonis is a sesquiterpene lactone with potent anti-inflammatory and antitumor activity [20]. 


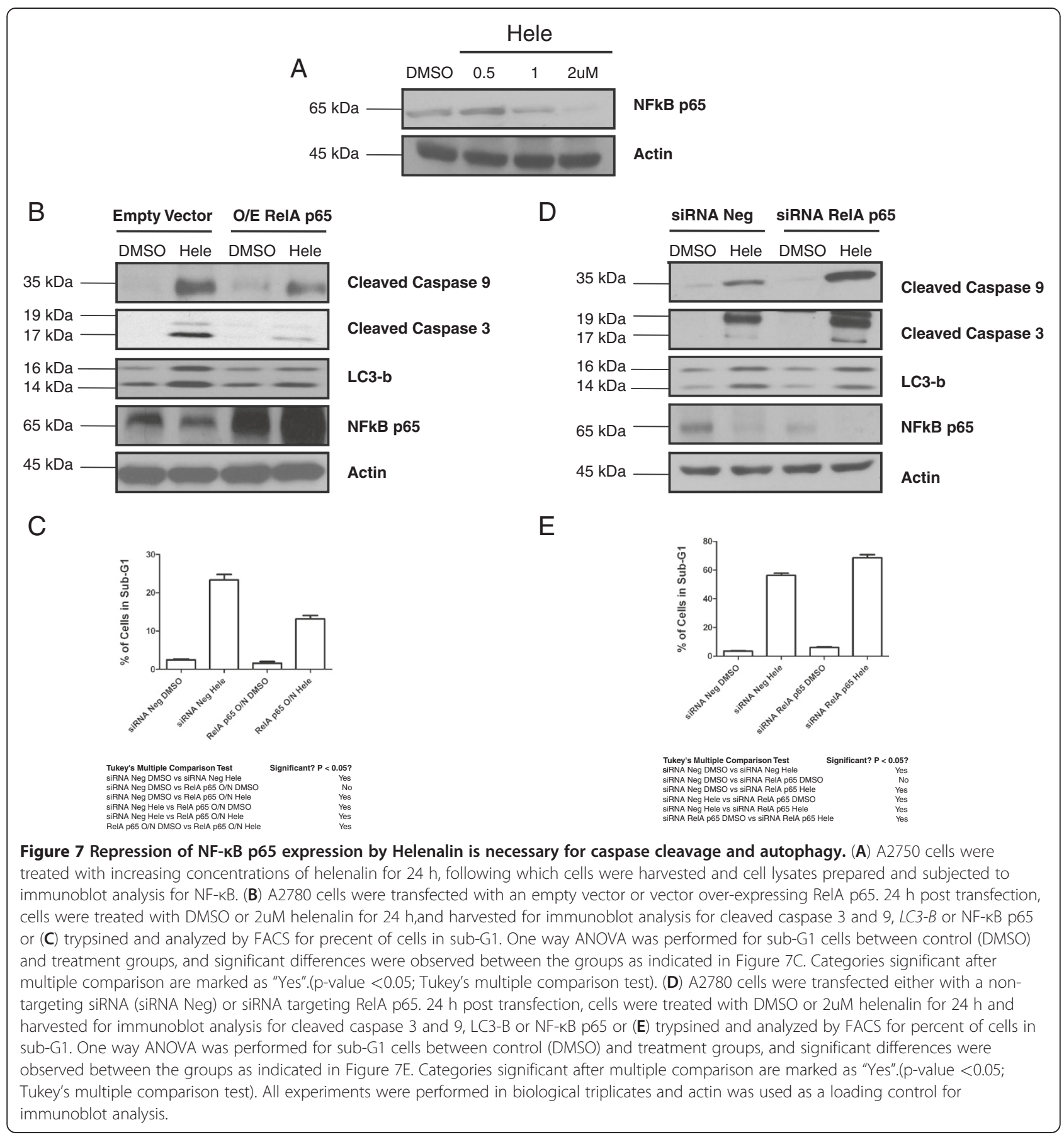

The use of helenalin has been demonstrated to reduce the growth of Staphylococcus aureus and Plasmodium falciparum [21] [22].In addition, previous studies have implicated helenalin to selectively inhibit the transcription factor NF-kB [7] and human telomerase activity [5], suggesting an underlying molecular mechanism for its antitumor activity.

Our ensuing findings derived from experiments performed in cancer cells treated with helenalin consistently resulted in an increase in cell death via apoptosis and autophagy. The increased sensitivity to cell death when exposed to helenalin was associated with increased levels of caspase cleavage. Indeed, when caspase cleavage was blocked using a specific inhibitor, cell death was considerably reduced. Given that several anticancer agents exert their effects by triggering autophagy, we postulated whether helenalin's action in triggering cell death was through the activation of autophagy. Treatment with helenalin resulted in an increase in defined autophagy markers, which when transcriptionally silenced using 
siRNA resulted in decreased cell death. Interestingly, transcriptionally silencing Atg12 and LC3-B, both essential for induction of autophagy cell death also resulted in a decrease of caspase activity. This result suggests that caspase activation is dependent on the expression of Atg12 and LC3-B. These observations are in agreement with previous studies where a decrease in LC3-B levels was associated with reduced autophagy and cells treated with LC3-B or Beclin 1 siRNA inhibited caspase-3/8 activation [13]. To further validate our findings, we performed Acridine Orange staining assays to measure acidic vesicular organelle (AVO) formation, a key indicator of autophagy initiation. AVO formation increased with increasing concentrations of helenalin and was suppressed with the use of bafilomycin A1, a highly potent and selective inhibitor of vacuolar $\mathrm{H}+-$ ATPases used in preventing the re-acidification of synaptic vesicles leading to the autophagy process [23-26].

We next examined whether helenalin's mechanism of action was through the transcription factor NF- $\kappa B$. Previous reports had revealed helenalin as a potent inhibitor of NF- $\mathrm{kB}$ [27] and that its binds with RelA disrupting its transcriptional activity [28]. In addition, NF- $\mathrm{kB}$ is a key regulator of several biological processes, including proliferation, differentiation, apoptosis and autophagy $[29,30]$. $\mathrm{NF}-\mathrm{KB}$ has been demonstrated to play an essential role after heat shock treatment by modulating autophagy by a mechanism to increase cell survival, possibly through the elimination of irreversibly damaged proteins [31-33]. With this regard, we observe that upon helenalin treatment, the level of NF-kB p65 (RelA) was reduced. Reintroducing RelA exogenously via an over-expression construct we observed that caspase activation was reduced together with the levels of autophagy markers, resulting in decreased cell death. Conversely, transcriptionally silencing NF- $\mathrm{kB}$ p65 had the outcome of increasing caspase cleavage, autophagy markers and cell death. These results strongly advocate the reliance of NF- $k B$ p 65 for helenalin induced autophagy cell death. We speculate helenalin downregulates NF- $\mathrm{KB}$ p65 expression via ubiquitination-mediated degradation. Previous reports have shown that tumor necrosis factor- $\alpha$ (TNF $\alpha$ ) polyubiquitinatates RelA at the lysine 195 residue which is critical for degradation of p65 [34]. The precise mechanism of p65 degradation needs to be further investigated.

In summary, we have shown that helenalin induces cell death via a mechanism involving the repression of NF-kB p65 expression resulting in an increase of autophagy markers and caspase activation. This provokes the clinically relevant question as to helenalin's use as a therapeutic intervention in patients with aberrant activation of NF-kB. Clinically, acute myeloid leukemia (AML) is an aggressive cancer with median survival rates of 2 to
3 months, and inhibition of NF- $\mathrm{KB}$ is considered one of the therapeutic strategies for treatment [30,35-39]. Oncogenic addiction of activated NF- $\mathrm{KB}$ could be inhibited with the use of helenalin, and as such could favorably be used in a therapeutic setting to augment tumor sensitivity to conventional chemotherapeutic drugs. Further work is necessary before helenalin can be considered as a lead compound and a treatment strategy. Specificity, toxicology, pharmacokinetics and metabolism needs to be investigated and studied further before it is introduced into the market.

\section{Additional files}

Additional file 1: Figure S1. Helenalin induces cell cycle arrest in G1
phase and modulates cell survival in a dose-dependent manner. (A)
MCF-7 cells were seeded onto 6-well plates and incubated for 24 h.
Various concentrations of helenalin were added to the culture medium
and incubated for an additional $24 \mathrm{~h}$. Cells were then harvested and
analyzed by flow cytometry. The cell cycle phase distribution was
determined using CellQuest software.(B) Percent of viable MCF-7 cells
after treatment with increasing concentrations of helenalin (serial dilution
for drug concentration ranging from 10uM to 0.001 MM) using the MTT
assay. (C) RKO cells were seeded onto 6-well plates and incubated for
24 h. Various concentrations of helenalin were added to the culture
medium and incubated for an additional 24 h. Cells were then harvested
and analyzed by flow cytometry. The cell cycle phase distribution was
determined using CellQuest software.(D) Percent of viable RKO cells after
treatment with increasing concentrations of helenalin (serial dilution for
drug concentration ranging from 10 uM to 0.001 uM) using the MTT assay.
All experiments were performed in biological triplicates.

\section{Competing interests}

The authors declare no competing financial interest and conflicts of interest with respect to the authorship and/or publication of this article.

\section{Acknowledgements}

This work was supported by the National Medical Research Council New Investigator Grant (NMRC/NIG/0036/2008), Singapore, to ZY.

\section{Author details}

${ }^{1}$ Division of Chemical Biology and Biotechnology, School of Biological Sciences, College of Science, Nanyang Technological University, 60 Nanyang Drive, Singapore 637551, Singapore. ${ }^{2}$ HeiLong Jiang University of Chinese Medicine, Harbin, People's Republic of China. ${ }^{3}$ National Cancer Centre of Singapore, NCCS-VARI Translational Research Laboratory, \#501, Level 5, 11 Hospital Drive, Singapore 169610, Singapore. ${ }^{4} 1$ stBASE Pte Ltd., 41 Singapore Science Park II, The Gemini, Singapore 117610, Singapore. ${ }^{5}$ MSD, Translational Medicine Research Center, 8 Biomedical Grove, \#04-01/-05 \& \#05-01/05, Neuros Building, Singapore 138665, Singapore.

\section{Authors' contributions}

N.K, P.Y.F, C.B.L and K.G.S carried out most of the experiments; C.B.L, M.S.H. and Z.Y. conceived and designed the project; J.L performed data analysis; M.S.H and Z.Y. interpreted the results and wrote the manuscript. All authors participated in data analysis. All authors read and edited the manuscript.

Authors' information

CBL and PYF are co-first authors.

Received: 20 January 2012 Accepted: 11 July 2012

Published: 11 July 2012

\section{References}

1. Paterson I, Anderson EA: Chemistry. The renaissance of natural products as drug candidates. Science 2005, 310(5747):451-453. 
2. Efferth $T$, Benakis A, Romero MR, Tomicic M, Rauh R, Steinbach D, Hafer R, Stamminger T, Oesch F, Kaina B, et al: Enhancement of cytotoxicity of artemisinins toward cancer cells by ferrous iron. Free Radic Biol Med 2004, 37(7):998-1009.

3. Efferth T, Rucker G, Falkenberg M, Manns D, Olbrich A, Fabry U, Osieka R: Detection of apoptosis in KG-1a leukemic cells treated with investigational drugs. Arzneimittelforschung 1996, 46(2):196-200.

4. Efferth T, Sauerbrey A, Olbrich A, Gebhart E, Rauch P, Weber HO, Hengstler $J G$, Halatsch ME, Volm M, Tew KD, et al: Molecular modes of action of artesunate in tumor cell lines. Mol Pharmacol 2003, 64(2):382-394.

5. Huang PR, Yeh YM, Wang TC: Potent inhibition of human telomerase by helenalin. Cancer Lett 2005, 227(2):169-174.

6. Berges C, Fuchs D, Opelz G, Daniel V, Naujokat C: Helenalin suppresses essential immune functions of activated CD4+ T cells by multiple mechanisms. Mol Immunol 2009, 46(15):2892-2901.

7. Lyss G, Knorre A, Schmidt TJ, Pahl HL, Merfort I: The anti-inflammatory sesquiterpene lactone helenalin inhibits the transcription factor NF-kappaB by directly targeting p65. J Biol Chem 1998, 273(50):33508-33516.

8. Zhao Y, Hamza MS, Leong HS, Lim CB, Pan YF, Cheung E, Soo KC, lyer NG: Kruppel-like factor 5 modulates p53-independent apoptosis through Pim1 survival kinase in cancer cells. Oncogene 2008, 27(1):1-8.

9. Traganos F, Darzynkiewicz Z: Lysosomal proton pump activity: supravital cell staining with acridine orange differentiates leukocyte subpopulations. Methods Cell Biol 1994, 41:185-194.

10. Nicoletti I, Migliorati G, Pagliacci MC, Grignani F, Riccardi C: A rapid and simple method for measuring thymocyte apoptosis by propidium iodide staining and flow cytometry. J Immunol Methods 1991, 139(2):271-279.

11. Telford WG, King LE, Fraker PJ: Comparative evaluation of several DNA binding dyes in the detection of apoptosis-associated chromatin degradation by flow cytometry. Cytometry 1992, 13(2):137-143.

12. Hoffmann R, von Schwarzenberg K, Lopez-Anton N, Rudy A, Wanner G, Dirsch VM, Vollmar AM: Helenalin bypasses Bcl-2-mediated cell death resistance by inhibiting NF-kappaB and promoting reactive oxygen species generation. Biochem Pharmacol 2011, 82(5):453-463.

13. Kim HP, Wang $X$, Chen ZH, Lee SJ, Huang MH, Wang Y, Ryter SW, Choi AM: Autophagic proteins regulate cigarette smoke-induced apoptosis: protective role of heme oxygenase-1. Autophagy 2008, 4(7):887-895

14. Telleria CM, Goyeneche AA, Stocco CO, Gibori G: Involvement of nuclear factor kappa $B$ in the regulation of rat luteal function: potential roles as survival factor and inhibitor of 20alpha-hydroxysteroid dehydrogenase. J Mol Endocrinol 2004, 32(2):365-383.

15. Copetti T, Bertoli C, Dalla E, Demarchi F, Schneider C: p65/RelA modulates BECN1 transcription and autophagy. Mol Cell Biol 2009, 29(10):2594-2608.

16. Newman DJ, Cragg GM: Natural products as sources of new drugs over the last 25 years. J Nat Prod 2007, 70(3):461-477.

17. Normile D: Asian medicine. The new face of traditional Chinese medicine. Science 2003, 299(5604):188-190.

18. Wiseman N: Traditional Chinese medicine: a brief outline. J Chem Inf Comput Sci 2002, 42(3):445-455.

19. Yuan $R$, Lin $Y$ : Traditional Chinese medicine: an approach to scientific proof and clinical validation. Pharmacol Ther 2000, 86(2):191-198.

20. Powis G, Gallegos A, Abraham RT, Ashendel CL, Zalkow LH, Grindey GB, Bonjouklian R: Increased intracellular $\mathrm{Ca} 2+$ signaling caused by the antitumor agent helenalin and its analogues. Cancer Chemother Pharmacol 1994, 34(4):344-350.

21. Boulanger D, Brouillette E, Jaspar F, Malouin F, Mainil J, Bureau F, Lekeux P: Helenalin reduces Staphylococcus aureus infection in vitro and in vivo. Vet Microbiol 2007, 119(2-4):330-338.

22. Francois G, Passreiter CM: Pseudoguaianolide sesquiterpene lactones with high activities against the human malaria parasite Plasmodium falciparum. Phytother Res 2004, 18(2):184-186.

23. Shacka JJ, Klocke BJ, Roth KA: Autophagy, bafilomycin and cell death: the "a-B-cs" of plecomacrolide-induced neuroprotection. Autophagy 2006, 2(3):228-230.

24. Shacka JJ, Klocke BJ, Shibata M, Uchiyama Y, Datta G, Schmidt RE, Roth KA: Bafilomycin A1 inhibits chloroquine-induced death of cerebellar granule neurons. Mol Pharmacol 2006, 69(4):1125-1136.

25. Wu YC, Wu WK, Li Y, Yu L, Li ZJ, Wong CC, Li HT, Sung JJ, Cho CH: Inhibition of macroautophagy by bafilomycin A1 lowers proliferation and induces apoptosis in colon cancer cells. Biochem Biophys Res Commun 2009, 382(2):451-456.

26. Yamamoto A, Tagawa Y, Yoshimori T, Moriyama Y, Masaki R, Tashiro Y: Bafilomycin A1 prevents maturation of autophagic vacuoles by inhibiting fusion between autophagosomes and lysosomes in rat hepatoma cell line, H-4-II-E cells. Cell Struct Funct 1998, 23(1):33-42.

27. Jin F, Liu X, Zhou Z, Yue P, Lotan R, Khuri FR, Chung LW, Sun SY: Activation of nuclear factor-kappaB contributes to induction of death receptors and apoptosis by the synthetic retinoid CD437 in DU145 human prostate cancer cells. Cancer Res 2005, 65(14):6354-6363.

28. Buchele B, Zugmaier W, Lunov O, Syrovets T, Merfort I, Simmet T: Surface plasmon resonance analysis of nuclear factor-kappaB protein interactions with the sesquiterpene lactone helenalin. Anal Biochem 2010, 401(1):30-7

29. Reikvam H, Olsnes AM, Gjertsen BT, Ersvar E, Bruserud O: Nuclear FactorkappaB Signaling: A Contributor in Leukemogenesis and a Target for Pharmacological Intervention in Human Acute Myelogenous Leukemia. Crit Rev Oncog 2009, 15(1):1-36.

30. Fabre C, Carvalho G, Tasdemir E, Braun T, Ades L, Grosjean J, Boehrer S, Metivier D, Souquere S, Pierron G, et al: NF-kappaB inhibition sensitizes to starvation-induced cell death in high-risk myelodysplastic syndrome and acute myeloid leukemia. Oncogene 2007, 26(28):4071-4083.

31. Djavaheri-Mergny M, Amelotti M, Mathieu J, Besancon F, Bauvy C, Codogno P: Regulation of autophagy by NFkappaB transcription factor and reactives oxygen species. Autophagy 2007, 3(4):390-392.

32. Nivon M, Richet E, Codogno P, Arrigo AP, Kretz-Remy C: Autophagy activation by NFkappaB is essential for cell survival after heat shock. Autophagy 2009, 5(6):766-783.

33. Xiao G: Autophagy and NF-kappaB: fight for fate. Cytokine Growth Factor Rev 2007, 18(3-4):233-243.

34. Fan $Y$, Mao R, Zhao $Y$, Yu Y, Sun W, Song P, Shi Z, Zhang D, Yvon E, Zhang $\mathrm{H}$, et al: Tumor necrosis factor-alpha induces RelA degradation via ubiquitination at lysine 195 to prevent excessive nuclear factor-kappaB activation. J Biol Chem 2009, 284(43):29290-29297.

35. Baumgartner B, Weber M, Quirling M, Fischer C, Page S, Adam M, Von Schilling C, Waterhouse C, Schmid C, Neumeier D, et al: Increased IkappaB kinase activity is associated with activated NF-kappaB in acute myeloid blasts. Leukemia 2002, 16(10):2062-2071.

36. Braun T, Carvalho G, Coquelle A, Vozenin MC, Lepelley P, Hirsch F, Kiladjian JJ, Ribrag V, Fenaux P, Kroemer G: NF-kappaB constitutes a potential therapeutic target in high-risk myelodysplastic syndrome. Blood 2006, 107(3):1156-1165.

37. Cilloni D, Messa F, Rosso V, Arruga F, Defilippi I, Carturan S, Catalano R, Pautasso M, Panuzzo C, Nicoli P, et al: Increase sensitivity to chemotherapeutical agents and cytoplasmatic interaction between NPM leukemic mutant and NF-kappaB in AML carrying NPM1 mutations. Leukemia 2008, 22(6):1234-1240.

38. Frelin C, Imbert V, Griessinger E, Peyron AC, Rochet N, Philip P, Dageville C, Sirvent A, Hummelsberger M, Berard E, et al: Targeting NF-kappaB activation via pharmacologic inhibition of IKK2-induced apoptosis of human acute myeloid leukemia cells. Blood 2005, 105(2):804-811.

39. Itoh M, Fu L, Tohda S: NF-kappaB activation induced by Notch ligand stimulation in acute myeloid leukemia cells. Oncol Rep 2009, 22(3):631-634

\section{doi:10.1186/1472-6882-12-93}

Cite this article as: Lim et al:: NF-KB p65 repression by the sesquiterpene lactone, Helenalin, contributes to the induction of autophagy cell death. BMC Complementary and Alternative Medicine 2012 12:93 